\title{
Prognostic and proliferative evaluation of ameloblastoma based on radiographic boundary
}

\author{
$\mathrm{Yi} \mathrm{Li}^{1,2}, \mathrm{Bo} \mathrm{Han}^{1}$ and Long-Jiang $\mathrm{Li}^{1,2}$
}

Ameloblastoma is a benign odontogenic tumor with an aggressive biological behavior, and the surgical treatment frequently results in failure for the postoperative recurrence. The aim of this article was to investigate whether the proliferative ability and prognosis of ameloblastoma could be evaluated by the radiographic boundary. The ameloblastoma cases treated by the conservative therapy in our hospital between 1981 and 2001 were divided into three groups based on the nature of the radiographic borders of the lesions. The biologic behavior was evaluated by $\mathrm{Ki}-67$ antibody immunohistochemically. Comparisons of prognosis and Ki-67 expression were carried out by statistic methods. There were 24 cases of well-defined edge with sclerosis (group I), 41 cases of well-defined edge without sclerosis (group II) and 32 cases of ill-defined edge (group III). The recurrent rates were $29.2 \%$ in group I, $43.9 \%$ in group II and $62.5 \%$ in group III $(P<0.05)$. The cells in group III expressed the highest Ki-67 level $(P<0.05)$. The radiographic boundary could be used as one of indicators in evaluating the proliferative ability of ameloblastoma and the patient's prognosis, which was consistent with Ki-67 expression.

International Journal of Oral Science (2012) 4, 30-33; doi:10.1038/ijos.2012.8; published online 24 February 2012

Keywords: ameloblastoma; Ki-67; radiography; prognosis

\section{INTRODUCTION}

Ameloblastoma is a benign odontogenic tumor with an aggressive biological behavior, ${ }^{1-2}$ and the surgical treatment frequently results in failure for the postoperative recurrence. Most authors deemed that its biological behavior could be predicted by the histological type, ${ }^{2-3}$ which could not be determined preoperatively. A biopsy performed before the surgery might bring patients additional injuries, and it is even hard for us to get enough tissues to make the histopathological diagnosis when the tumors were embedded deeply in the bone. Therefore, the radiographic examination, as a trauma-free auxiliary method, is important and helpful in evaluating the biological behavior of the tumor. ${ }^{4}$ It is possible to estimate growth rate by the radiographic image because we could get information from radiographs including the bone destruction, the reactions of bone, the edge of lesion and other details such as location and size of the tumor. ${ }^{5}$

There was much research of ameloblastoma carried out previously, such as histological types and the treatments. ${ }^{2,6-7}$ It was found that ameloblastomas of different radiographic appearances had not similar biological behaviors. ${ }^{6}$ But there was not a research to analyze the proliferative ability and prognosis of the ameloblastoma based on its radiographic boundary.

We hypothesized that ameloblastoma with an ill-defined radiographic boundary had a higher proliferative ability, and when treated by the conservative therapy, the prognosis of patients with an ill-defined radiographic boundary was significantly worse than those with well-defined ones. We carried out the following experiment to test the hypothesis, and the proliferative ability of ameloblastoma was investigated by $\mathrm{Ki}-67$. The aim of this article was to better understand the lesion and its radiographic features, and to make patients get a better quality of life postoperatively.

\section{MATERIALS AND METHODS}

Patients

We studied the ameloblastoma cases of the last 20 years retrieved from the files of West China Stomatology Hospital of Sichuan University between 1981 and 2001 and focused on the patients who received a conservative treatment. Based on the third edition of the World Health Organization's histological classification, ${ }^{8}$ the pathological diagnoses were confirmed with the hematoxylin-eosin slides by the pathologists. All cases had integrated clinical data and had been followed up for more than 48 months. This study was approved by our Institutional Review Board. The Declaration of Helsinki protocols were followed during the whole study.

\section{Radiographs}

Panoramics of all patients were reviewed as the basic method to evaluate the radiographic boundaries and gain other information such as the size and location of the lesions. If necessary, computer tomography was employed to assist in providing the information in the buccolingual direction. The patients receiving a conservative treatment were

\footnotetext{
${ }^{1}$ State Key Laboratory of Oral Diseases, Sichuan University, Chengdu, China and ${ }^{2}$ Department of Head and Neck Oncology, West China College of Stomatology, Sichuan University, Chengdu, China

Correspondence: Professor LJ Li, State Key Laboratory of Oral Diseases, Sichuan University; Department of Head and Neck Oncology, West China College of Stomatology, Sichuan University, Chengdu 610041, China

E-mail: muzili63@163.com

Received 26 January 2011; Accepted 16 September 2011
} 

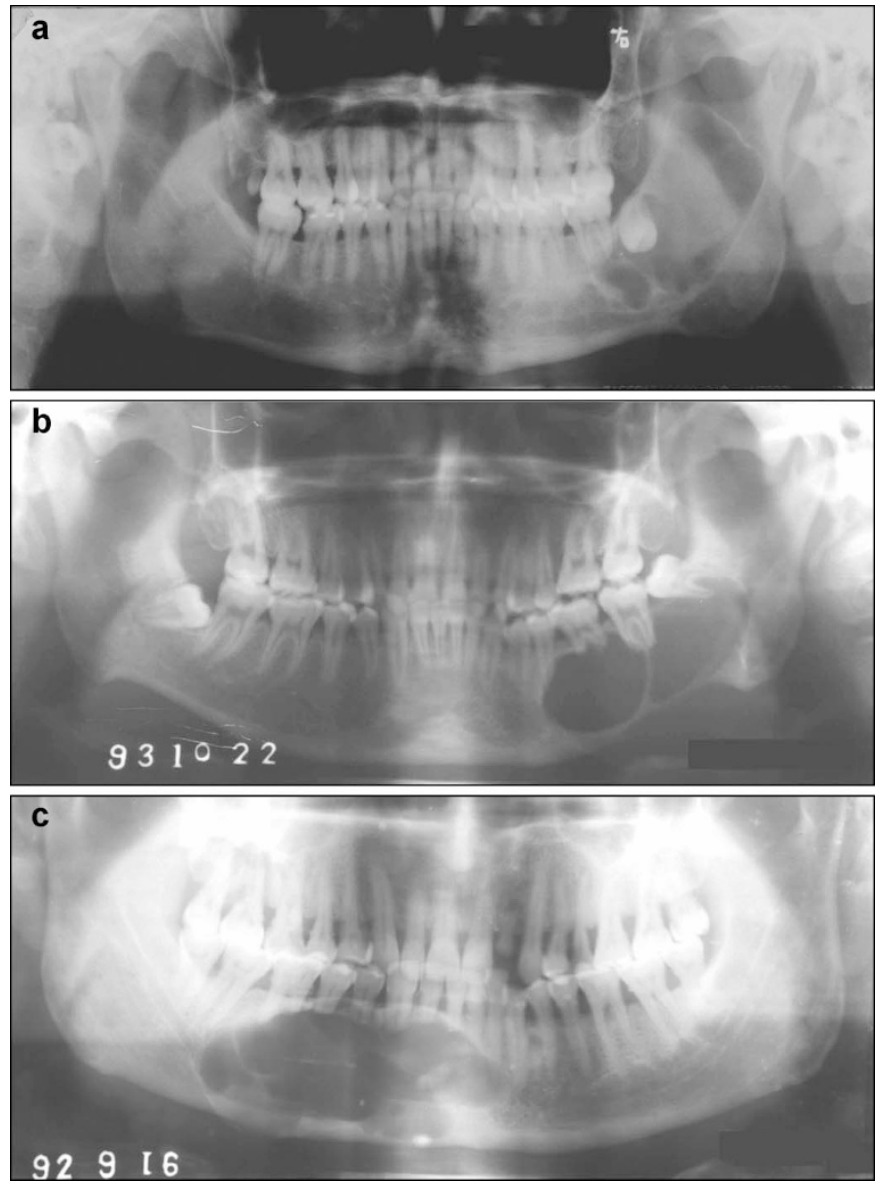

Figure 1 The radiographs of the different groups. (a) Group I: well-defined edge with sclerosis; (b) group II: well-defined edge without sclerosis; (c) group III: illdefined edge.

divided into three groups according to the nature of the lesions' radiographic boundaries: group I: well-defined with sclerosis (Figure 1a); group II: well-defined without sclerosis (Figure 1b); group III: illdefined (Figure 1c). The radiograph of group I presented a confined radiolucent lesion with a sharp edge and a sclerotic line existing in more than $80 \%$ of the border between the normal bone and the lesion. The radiograph of group II was a confined radiolucent lesion with a sharp edge which was similar to that of group I, but with a sclerotic line existing in less than $20 \%$ of the border. If any part of the edge was not well-demarcated, the case was assigned into group III.

We invited one radiologist and one oral and maxillofacial surgeon to classify the lesions. Both the observers had more than 10 years of experiences in oral radiology and did not know the recurrence status when they classified the cases. They were standardized before the present research. In case of disagreement between observers, the classification was done by a consultation.

\section{Immunohistochemistry}

The proliferative ability of the tumor was investigated by Ki-67 monoclonal antibody (clone MIB-1; Dako, Glostrup, Denmark) immunohistochemically, which were performed in a standard avidin-biotin immunoperoxidase procedure. Two pathologists, who both had more than 10 years of experience in immunohistochemistry and did not know the recurrence status of the cases, were invited to evaluate Ki-67 expression. Each of them used their own eyepiece graticule. The label index (LI) of Ki-67 was evaluated by the proportion of positive cells in all tumor cells in 10 adjacent high-power fields $(\times 40)$.

\section{Statistical analysis}

Using the software of SPSS13.0 (SPSS, Chicago, IL, USA), comparison of prognosis and radiological features between the three groups was carried out by Chi-square test, and the mean value of Ki-67 LI was tested by Student's $t$-tests. $P$ values below 0.05 were considered significant. The inter- and intra-observer reliabilities of the observers of radiographs or immunohistochemistry were tested by Kappa test.

\section{RESULTS}

The medical files of 178 ameloblastoma cases were reviewed in this study. Ninety-seven patients received a conservative treatment, which was the curettage or enucleation of the tumor and the periodic followup. Their ages ranged from 21 to 63 years (37.2 \pm 8.6 year). There were 51 male and 46 female patients with a ratio of $1.11: 1$. All the lesions were located in the mandible and presented as unilocular or multilocular radiolucent regions. The differences of radiological features were not statistically significant $(P>0.05)$.

The results showed that the prognoses were different in patients receiving a radical surgery or a conservative one: $46.4 \%$ of the patients receiving a conservative treatment suffered recurrence (45 of 97), and only $1.2 \%$ of the patients receiving a radical surgery experienced recurrence ( 1 of 81 ).

Table 1 showed the prognoses of patients treated by the conservative surgery, who were divided into the three groups of different radiographic boundaries. These 97 cases consist of 24 cases of well-defined edge with sclerosis (group I), 41 ones of well-defined edge without sclerosis (group II) and 32 ones of ill-defined edge (group III). In the grouping procedure, the consistency of observers was satisfying, and the Kappa value was higher than 0.80 . There were seven cases in group I recurring in 18-39 months (26.4 \pm 8.3 months) with the recurrent rate of $29.2 \%$ ( 7 of 24 ), 18 cases in group II recurring in 12-36 months

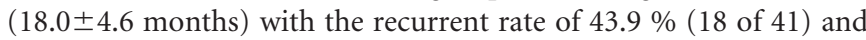
20 cases in group III recurring in 6-42 months ( $12.0 \pm 5.4$ months) with the recurrent rate of $62.5 \%$ ( 20 of 32 ). The statistic difference was considered significant among the three groups $(P<0.05)$.

Table 1 Results of the conservative management and the expression of $\mathrm{Ki}-67$ antigen

\begin{tabular}{|c|c|c|c|c|c|c|}
\hline \multirow[b]{2}{*}{ Group } & \multirow[b]{2}{*}{$N$} & \multicolumn{2}{|c|}{ Results of therapy } & \multicolumn{2}{|c|}{$\mathrm{LI}$ of $\mathrm{Ki}-67$ antigen } & \multirow[b]{2}{*}{ Recurrent rate/\% } \\
\hline & & Tumor-free & Recurrence & Range/\% & Mean \pm s.d. $/ \%$ & \\
\hline Group ${ }^{a}$ & 24 & 17 & 7 & $0.94-4.06$ & $2.06 \pm 0.82$ & 29.2 \\
\hline Group $\|^{a}$ & 41 & 23 & 18 & $1.93-7.42$ & $3.17 \pm 1.23$ & 43.9 \\
\hline Group III & 32 & 12 & 20 & $1.06-8.43$ & $4.25 \pm 1.01$ & 62.5 \\
\hline
\end{tabular}

Abbreviation: LI, label index.

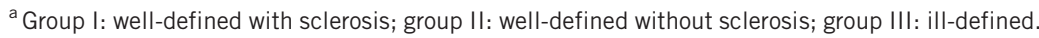



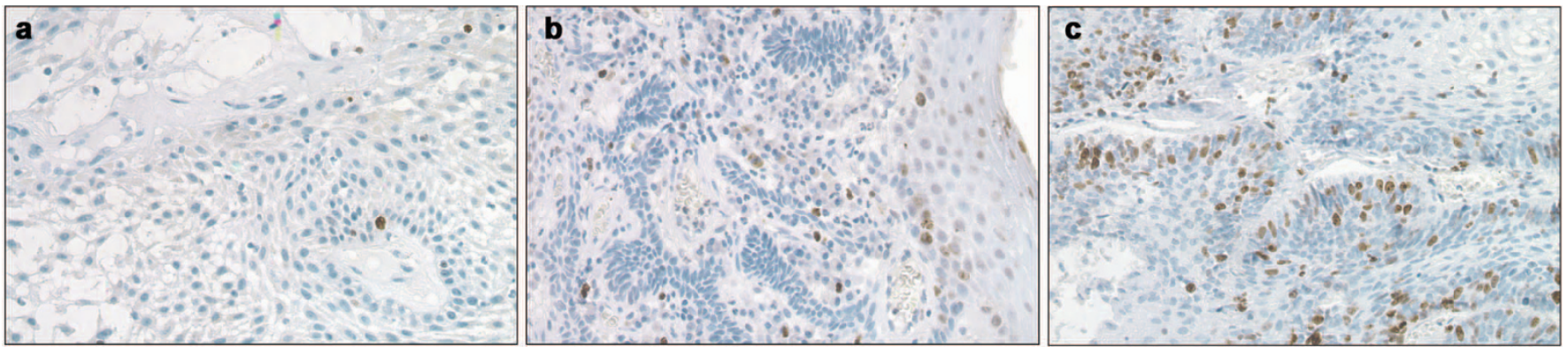

Figure 2 The immunohistochemical staining of the three groups (×200). (a) Group I: well-defined edge with sclerosis; (b) group II: well-defined edge without sclerosis; (c) group III: ill-defined edge.

In the immunohistochemical study, there were statistic difference in the expressions of Ki-67 (Figure 2) among all the three groups $(P<0.05)$, and so were in the average LI. The results showed that the ameloblastoma with an ill-defined radiographic boundary had the highest percentage of Ki-67-positive cells among the three groups (Table 1). The Kappa values of observers were higher than 0.85 .

\section{DISCUSSION}

The management of ameloblastoma places the oral and maxillofacial surgeons in a dilemma whether to take a conservative or a radical treatment. For a long time, surgeons deemed that the ameloblastoma was a benign odontogenic neoplasm, and could be treated by the curettage or enucleation. If the tumor recurred, it could be treated with a secondary surgery in a smaller region than the original lesion. ${ }^{9-10}$ Although it is considered a benign tumor, ameloblastoma has aggressive behaviors including local recurrence, cancerization or even distant metastasis. Therefore, many surgeons tended to take a radical surgery when facing this disease. Our research showed that the prognosis of ameloblastoma was associated mainly with the method of surgical treatment, which meant that patients receiving a conservative treatment had a worse prognosis than those who received a radical one. It seemed that the best way for treating the ameloblastoma was extensive resection of the tumor with a wide region in the normal bone. ${ }^{3,11}$

We believe that the radical surgery is a best choice for many patients of ameloblastoma, but not for all. Our data showed that more than $50 \%$ patients receiving the conservative treatment had good prognosis without any recurrence. In another word, more than $50 \%$ patients could be treated by a conservative method, and maintain the continuity of their jaws to have a better quality of life. Obviously, the radical surgery is an overtreatment if we chose it as a routine way. How to estimate the recurrent probability is the key point to establish a rational treatment plan.

Many factors were related with the prognosis of ameloblastoma. Some scholars ${ }^{12-13}$ believed that a radical surgery should be used for the multicystic ameloblastoma to prevent the recurrence. From the pathological aspect, the follicular ameloblastomas were thought to have a higher recurrence rate than plexiform or unicystic. ${ }^{14}$ Takahashi et al. ${ }^{9}$ found that ameloblastoma in children was mainly the plexiform type, and conservative treatment could be accepted as the initial treatment. Due to lack of the cortical plates, ameloblastoma in maxilla was thought to spread readily into the adjacent vital regions and suggested to be treated by extensive resection. ${ }^{14-15}$

It is valid to use radiographs to estimate the growth rate of focal osseous lesions in clinical practice. Ueno et al. ${ }^{6}$ reported that biological behavior of the ameloblastoma was related to the radiographic appearance, and the multilocular type of ameloblastoma had a poor prognosis. The radiographic boundary of ameloblastoma is another useful parameter in evaluating the growth rate of the tumor. Kramer ${ }^{16}$ stated that, while ameloblastomas invaded the intertrabecular spaces of cancellous bone, they do not invade compact bone, although they may erode it. Ameloblastoma which has a well-defined edge with sclerosis is thought to grow slowly, and the normal bone has a strong reaction to form the sclerosis edge, which acts like compact bone to resist the invasion of the tumor, even if the size of the lesion is comparably large. So the tumor is confined and the prognosis is good. On the contrary, if the tumor's radiographic boundary is not sclerotized, the tumor was thought to be a little more aggressive and the prognosis is not so optimistic. Ameloblastomas with ill-defined boundary are thought to have the most aggressive behavior than others, which should be treated by the radical surgery because of the higher recurrent probability.

The accurate evaluation of radiographic edges was the key point in this study, so we invited two experts who had abundant experiences in the radiographic diagnosis to group the cases as objectively as possible. It was based on the clinical experiments of our department for over 40 years that we chose $80 \%$ of sclerotic line as the standard to divided patients of groups I and II. And the results confirmed its efficiency. But we had to admit that it was comparatively subjective. With the development of the digital technology, we could use digital radiographic system to evaluate the radiographic boundary between normal bone and lesions more objectively, and with further research on the relationship between the biological behavior and radiological boundary, we expect to gain more and objective results in the further studies.

Proliferation is a key feature of the progression of tumors. It was found that the proliferating activity of each type of ameloblastoma can be indexed by the expression of Ki-67 antigen. ${ }^{7,17-18}$ We found that the $\mathrm{Ki}-67 \mathrm{LI}$ of the ameloblastoma in group III was the highest among the three groups which could be attributed to the fact that ameloblastoma with ill-defined radiographic boundary contained more cells with higher proliferative ability. This result was consistent with the retrospective analysis of clinical data. With the ill-defined radiographic boundary, the tumor has the highest proliferative ability and poorest prognosis.

\section{CONCLUSION}

In our article, we raised a hypothesis that that the radiographic boundary of ameloblastoma was related to the tumor's proliferative ability and the patient's prognosis. In order to testify this hypothesis, we carried out a retrospective analysis of the ameloblatoma cases in our hospital and an experiment of $\mathrm{Ki}-67$ expression in these cases. 
Although the recurrent rate was relatively high in the group I, the results could support the hypothesis. It means that we could use the radiographic boundary as a reference to the patients for choosing surgical plan, apart from the lesion size, location, patient age, clinical and pathological types, and other parameters, and avoid the overtreatment to make those patients get a better quality of life.

\section{ACKNOWLEDGEMENTS}

We would like to thank Mr Gavin McCloskey for his kind help on the English editing of this article.

1 Laughlin EH. Metastasizing ameloblastoma. Cancer 1989; 64(3): 776-780.

2 Reichart PA, Philipsen HP, Sonner S. Ameloblastoma: biological profile of 3677 cases. Eur J Cancer 1995; 31B(2): 86-99.

3 Gardner DG. A pathologist's approach to the treatment of ameloblastoma. J Oral Maxillofac Surg 1984; 42(3): 161-166.

4 O'Donnell. Evaluation of focal bone lesions: basic principles and clinical scenarios. Imaging 2003; 15(4): 298-323.

5 Lodwick GS, Wilson AJ, Farrell $\mathrm{C}$ et al. Determining growth rates of focal lesions of bone from radiographs. Radiology 1980; 134(3): 577-583.

6 Ueno S, Mushimoto K, Shirasu R. Prognostic evaluation of ameloblastoma based on histologic and radiographic typing. J Oral Maxillofac Surg 1989; 47(1): 11-15.

7 Meer S, Galpin JS, Altini M et al. Proliferating cell nuclear antigen and Ki67 immunoreactivity in ameloblastomas. Oral Surg Oral Med Oral Pathol Oral Radiol Endod 2003; 95(2): 213-221.
8 Gardner DG, Heikinheimo K, Shear M et al. Ameloblastomas. In: Barnes L, Eveson JW, Reichart P, Sidransky D (ed.) World Health Organization Classification of Tumors. Pathology \& Genetics of Head and Neck Tumours. Lyon: IARC Press, 2005: 296-300.

9 Takahashi K, Miyauchi K, Sato K. Treatment of ameloblastoma in children. Br J Oral Maxillofac Surg 1998; 36(6): 453-456.

10 Sammartino G, Zarrelli C, Urciuolo V et al. Effectiveness of a new decisional algorithm in managing mandibular ameloblastomas: a 10-years experience. Br J Oral Maxillofac Surg 2007; 45(4): 306-310.

11 Hong J, Yun PY, Chung IH et al. Long-term follow up on recurrence of 305 ameloblastoma cases. Int J Oral Maxillofac Surg 2007; 36(4): 283-288.

12 Muller H, Slootweg PJ. The ameloblastoma, the controversial approach to therapy. $J$ Maxillofac Surg 1985; 13(2): 79-84.

13 Rapidis AD, Andressakis DD, Stavrianos SD et al. Ameloblastomas of the jaws: clinicopathological review of 11 patients. Eur J Surg Oncol 2004; 30(9): 998-1002.

14 Gardner DG, Pecak AM. The treatment of ameloblastoma based on pathologic and anatomic principles. Cancer 1980; 46(11): 2514-2519.

15 Sehdev MK, Huvos AG, Strong EW et al. Proceedings: ameloblastoma of maxilla and mandible. Cancer 1974; 33(2): 324-333.

16 Kramer IR. Ameloblastoma: a clinicopathological appraisal. Br J Oral Surg 1963; 1: 13-28.

17 Sandra F, Mitsuyasu T, Nakamura N et al. Immunohistochemical evaluation of PCNA and Ki-67 in ameloblastoma. Oral Oncol 2001; 37(2): 193-198.

$18 \mathrm{Han} \mathrm{B}$, Li L, Wang H. Expression of Ki-67 antigen in ameloblastoma and its clinical significance. Hua Xi Kou Qiang Yi Xue Za Zhi 2003; 21(2): 153-154. Chinese.

cc This work is licensed under a Creative Commons OMERIGHISHESERVED Attribution-NonCommercial-NoDerivative Works 3.0 Unported License. To view a copy of this license, visit http:// creativecommons.org/licenses/by-nc-nd/3.0 